\title{
Methylenetetrahydrofolate reductase gene polymorphisms and lung cancer: a meta-analysis
}

\author{
Renfang Mao $\cdot$ Yihui Fan $\cdot$ Yan Jin · \\ Jing Bai $\cdot$ Songbin Fu
}

Received: 9 November 2007/ Accepted: 22 January 2008/Published online: 14 March 2008

(C) The Japan Society of Human Genetics and Springer 2008

\begin{abstract}
So far, case-control studies on the association between methylenetetrahydrofolate reductase (MTHFR) gene polymorphisms and lung cancer provide controversial or inconclusive results. To clarify the effect of MTHFR polymorphisms on the risk of lung cancer, a meta-analysis of all case-control observational studies was performed. The studies provided 5,111/6,415 cases/controls for C677T and 5,087/6,232 cases/controls for A1298C. The heterogeneity $\left(P=0.0001, I^{2}=76.6 \%\right)$ for C677T among the eight studies was extreme. Cluster analyses based on the frequencies of the C677T genotype of the control group in each study revealed that the studies could not cluster together according to their ethnicity. The random effects (RE) model showed that the 677T allele was not associated with the risk of lung cancer compared with the $\mathrm{C}$ allele $[\mathrm{OR}=1.12,95 \%$ confidence interval (CI) (0.97-1.28), $P=0.12]$. The contrast of homozygotes, recessive model, dominant model produced the same pattern of results as the allele contrast. In regard to the A1298C polymorphism, there was no heterogeneity among the seven studies comparing the $\mathrm{C}$ versus the A allele $\left(P=0.24, I^{2}=24.4 \%\right)$, but no significant association was detected by the RE model or the fixed effects model [FE odds ratio $(\mathrm{OR})=$ $0.99(0.93-1.05)$ and $\mathrm{RE} \mathrm{OR}=1.00(0.92-1.08)]$. The
\end{abstract}

R. Mao · Y. Fan · Y. Jin · J. Bai · S. Fu ( $₫)$

Laboratory of Medical Genetics, Harbin Medical University,

Harbin 150081, China

e-mail: fusb@ems.hrbmu.edu.cn

S. Fu

Bio-pharmaceutical Key Laboratory of Heilongjiang Province, Harbin 150081, China effect of MTHFR polymorphisms (C677T, A1298C) on the risk of lung cancer was undetectable, even though analyzed on a relatively good number of subjects (totally 11,526 subjects) by meta-analysis (statistical power $=93.9 \%$ ). Although MTHFR polymorphisms were associated with the risk of colorectal cancer, leukemia, and gastric cancer supported by other meta-analysis, our pooled data suggest no evidence for a major role of these two variants in carcinogenesis of lung cancer. The results implied that different tumors evolve by different pathological pathways and the roles of MTHFR in cancer is determined by its target genes.

Keywords MTHFR polymorphisms - Lung cancer . Meta-analysis

\section{Introduction}

Lung cancer is the leading cause of cancer death worldwide. Despite improvements in its treatment in recent years, the prognosis from this disease has only marginally improved, with 5-year survival rates for both small (SCLC) and non-small-cell lung cancer (NSCLC) typically being no better than $15 \%$ (Jemal et al. 2002). Therefore, efforts toward primary prevention in addition to early detection have come under the spotlight.

As the predominant risk factor for lung cancer, smoking [relative risk $(\mathrm{RR})=10-30$ compared with nonsmokers (American Cancer Society; Centers for Disease Control and Prevention)] is directly linked to lung cancer in $90 \%$ of women and $79 \%$ of men (Bilello et al. 2002). Secondhand smoke exposure is also a risk factor (Fontham et al. 1994; Environmental Protection Agency). However, appreciation that the development of most cancers results from a 
complex interaction between both environmental and genetic factors is growing. It now seems likely that heritable factors are of significant importance in lung cancer independent of smoking history or exposure to environmental tobacco smoke (Minna et al. 2002; Schwartz et al. 2006; Amos et al. 1999).

Many epidemiological studies have provided evidence that high consumption of vegetables and fruits is associated with a reduced risk of lung cancer (Steinmetz et al. 1991; Takezaki et al. 2001). Folate is one of the constituents found in vegetables and fruits, and dietary folate may be one of the micronutrients that provide protection against lung carcinogenesis (Bandera et al. 1997; Voorrips et al. 2000; Shen et al. 2003). Biological functions of folate within so-called one-carbon metabolism are to facilitate de novo deoxynucleoside triphosphate synthesis and to provide methyl groups required for intracellular methylation reactions. Methylenetetrahydrofolate reductase (MTHFR), a pivotal enzyme in folate and homocysteine metabolism, catalyzes irreversibly the reduction of 5,10-methylenetetrahydrofolate $\left(\mathrm{CH}_{2}-\mathrm{THF}\right)$ to 5-methylenetetrahydrofolate $\left(\mathrm{CH}_{3}-\mathrm{THF}\right)$. Thus, MTHFR might play roles in the etiology of lung cancer in combination with environmental factors such as folate consumption.

There are many reports that the MTHFR singlenucleotide polymorphisms (SNPs) C677T and A1298C are associated with many diseases, e.g., coronary artery disease (Kluijtmans et al. 1997), stroke (Banerjee et al. 2007), colorectal cancer (Hubner et al. 2007), etc. To our knowledge, Shen et al. first examined the association between the MTHFR gene polymorphisms and risk of lung cancer and suggested that the MTHFR C677T and A1298C polymorphisms did not play an important role in the etiology of lung cancer (Shen et al. 2001). However, Jeng et al. pointed out in 2003 that the MTHFR C677T variant genotype did reduce the risk of lung cancer (Jeng et al. 2003). The same year, Siemianowicz et al. reported lung cancer patients had statistically higher percentage of MTHFR 677TT genotype in comparison with noncancer controls (Siemianowicz et al. 2003). In 2007, two papers also got the distinct results (Suzuki et al. 2007; Hung et al. 2007). As we know from case-control studies, there are possibilities that some positive results might be spurious and some negative findings might be a consequence of low statistical power because of the study's small sample size or methodological shortcomings (Hassan and Markus 2000; Casas et al. 2004; Dichgans and Markus 2005). Meta-analysis is a powerful means of resolving disparate results. To clarify the effect of MTHFR polymorphisms on the risk of lung cancer, a meta-analysis of all case-control observational studies was performed in this study.

\section{Methods}

Study identification and selection

To identify all articles that examined the association of MTHFR C677T and A1298C polymorphisms with lung cancer, we conducted a literature search of the PubMed database (before August 2007) using the following MeSH terms and Entry terms.

MeSH terms: methylenetetrahydrofolate reductase (NADPH2)

Entry terms: methylenetetrahydrofolate reductase MTHFR, methylene-THF reductase (NADPH), 5,10-methylenetetrahydrofolate reductase (NADPH), methylenetetrahydrofolate reductase (NADPH), methylene tetrahydrofolate reductase, tetrahydrofolate reductase, methylene

MeSH terms: lung neoplasms

Entry terms: neoplasms, pulmonary; neoplasm, pulmonary; pulmonary neoplasm; neoplasms, lung; lung neoplasm; neoplasm, lung; pulmonary neoplasms; lung cancer; cancer, lung; cancers, lung; lung cancers; cancer of lung; pulmonary cancer; cancer, pulmonary; cancers, pulmonary; pulmonary cancers; cancer of the lung

The retrieved publications were then read in their entirety to assess their appropriateness for inclusion in this metaanalysis. All references cited in the studies were also reviewed to identify additional published work not indexed by PubMed database. Abstracts, case reports, editorials, and review articles were excluded. Studies included in the meta-analysis had to meet all of the following criteria: (a) an unrelated case-control design was used (b) genotype frequency was available.

\section{Data extraction}

The following information was extracted from each study: first author, year of publication, ethnicity of study population, genotyping method, and the number of cases and controls for each C677T or A1298C genotype.

Statistical analysis

The meta-analysis examined the overall association for the allele contrast, the contrast of homozygotes, and the recessive and dominant models. The effect of association was indicated as odds ratio (OR) with the corresponding 95\% confidence interval (CI). The pooled OR was 
estimated using fixed effects (FE) (Mantel-Haenszel) and random effects (RE) (DerSimonian and Laird) models (Lau 1997). The heterogeneity between studies was tested using the $Q$ statistic (Zintzaras and Ioannidis 2005). The heterogeneity was considered statistically significant with $P<0.10$. Heterogeneity was quantified using the $I^{2}$ metric, which is independent of the number of studies in the meta-analysis $\left(I^{2}<25 \%\right.$ no heterogeneity; $I^{2}=25-50 \%$ moderate heterogeneity; $I^{2}>50 \%$ large or extreme heterogeneity) (Higgins and Thompson 2002). Publication bias was investigated by funnel plot, in which the standard error in $\log (\mathrm{OR})$ in each study was plotted against OR. An asymmetric plot suggested possible publication bias. Funnel plot asymmetry was assessed via Egger's linear regression test, a linear regression approach to measuring funnel plot asymmetry on the natural logarithm scale of the OR (Egger et al. 1997). The significance of the intercept was determined by the $t$ test as suggested by Egger et al., and a $P$ value of $<0.05$ was considered significant. HardyWeinberg equilibrium (HWE) was tested by the chi-square test. Clustering analysis and the dendrogram of the clustering results were carried out using the SPSS 10.0 program. Analyses were performed using the software ReviewManage 4.2.

\section{Results}

\section{Eligible studies}

To include all of the published articles, we used MeSH terms and Entry terms as the retrieve strategy. We found 26 published articles addressing MTHFR and lung cancer. However, data from only eight articles met the inclusion criteria, and seven of them also provide data on A1298C (Table 1). The studies were published between 2001 and 2007. In all studies, the cases were histologically confirmed and the controls were free of lung cancer and were matched for age and gender. For determination of the genetic polymorphisms of C677T and A1298C, validated genotyping methods were used in all studies: polymerase chain reaction restriction fragment length polymorphism (PCR-RFLP) (Shen et al. 2001; Jeng et al. 2003; Siemianowicz et al. 2003; Shi et al. 2005; Zhang et al. 2005); real-time PCR (Shen et al. 2005; Suzuki et al. 2007; Hung et al. 2007). Studies were conducted in various populations of different ethnicities: four were conducted in populations of East Asian ethnicity (Jeng et al. 2003; Shen et al. 2005; Zhang et al. 2005; Suzuki et al. 2007), two involved non-Hispanic whites (Shen et al. 2001; Shi et al. 2005), and two Europeans (Siemianowicz et al. 2003; Hung et al. 2007). Genotype distributions in the control population of one study (Zhang et al. 2005) significantly deviated from HWE (Tables 2, 3).
Hierarchical cluster analysis

A dendrogram of the clustering results among the eight studies was constructed by hierarchical cluster analysis using SPSS 10.0 software. It was based on the genotype frequency of the control group in every study. The results of cluster analysis might give a powerful evidence for the reliability of each study, because the control group should cluster together according to ethnicity. As shown in Fig.1, articles 1, 5, 8 (Shen et al. 2001; Shi et al. 2005; Hung et al. 2007) cluster together as European; articles 4, 6, 7 (Shen et al. 2005; Zhang et al. 2005; Suzuki et al. 2007) cluster together as East Asian ethnicity. Sensitivity analyses would also be performed according to the results of cluster analysis.

\section{Summary statistics}

The studies provided 5,111/6,415 cases/controls for C677T and 5,087/6,232 cases/controls for A1298C. The variant genotype and allele frequencies of C677T and A1298C in the individual studies are shown in Tables 2 and 3. Overall, for C677T polymorphism, the frequency of CC-homozygous individuals was $41.9 \%$; the frequency of CTheterozygous individuals was $44.6 \%$, and the frequency of TT-homozygous individuals was $13.4 \%$ in the case. In the control, the frequencies of CC-homozygous individuals, CT-heterozygous individuals, and TT-homozygous individuals were $44.8 \%, 43.3 \%$, and $11.9 \%$, respectively. The $677 \mathrm{~T}$ allelic frequency in the case and control was $35.8 \%$ and $33.5 \%$, respectively. The A allele was the most common for A1298C polymorphism, and the frequency of the AA genotype was the highest in both cases and controls, whereas that for genotype CC was the lowest (Table 3). The frequency of $1298 \mathrm{C}$ polymorphism in the case was $26.1 \%$, whereas in the control, it was $28.5 \%$. In one study (Zhang et al. 2005) of the A1298C polymorphism, the distribution of genotypes in the control group was not in HWE $(P<0.05)$, indicating genotyping errors and/or population stratification.

Main results, subgroup, and sensitivity analyses

The heterogeneity results and the effect of the association between polymorphisms of MTHFR and the risk of lung cancer for the genetic contrasts under investigation are shown in Figs. 2 and 3 and Table 4.

There was extreme heterogeneity $(P=0.0001$, $\left.I^{2}=76.6 \%\right)$ among the eight studies. To eliminate heterogeneity, we subgrouped the eight studies as far as possible (based on genotype method, ethnicity, control group, publication year, and so on), but the heterogeneity also existed. Cluster analysis based on the genotype frequency 


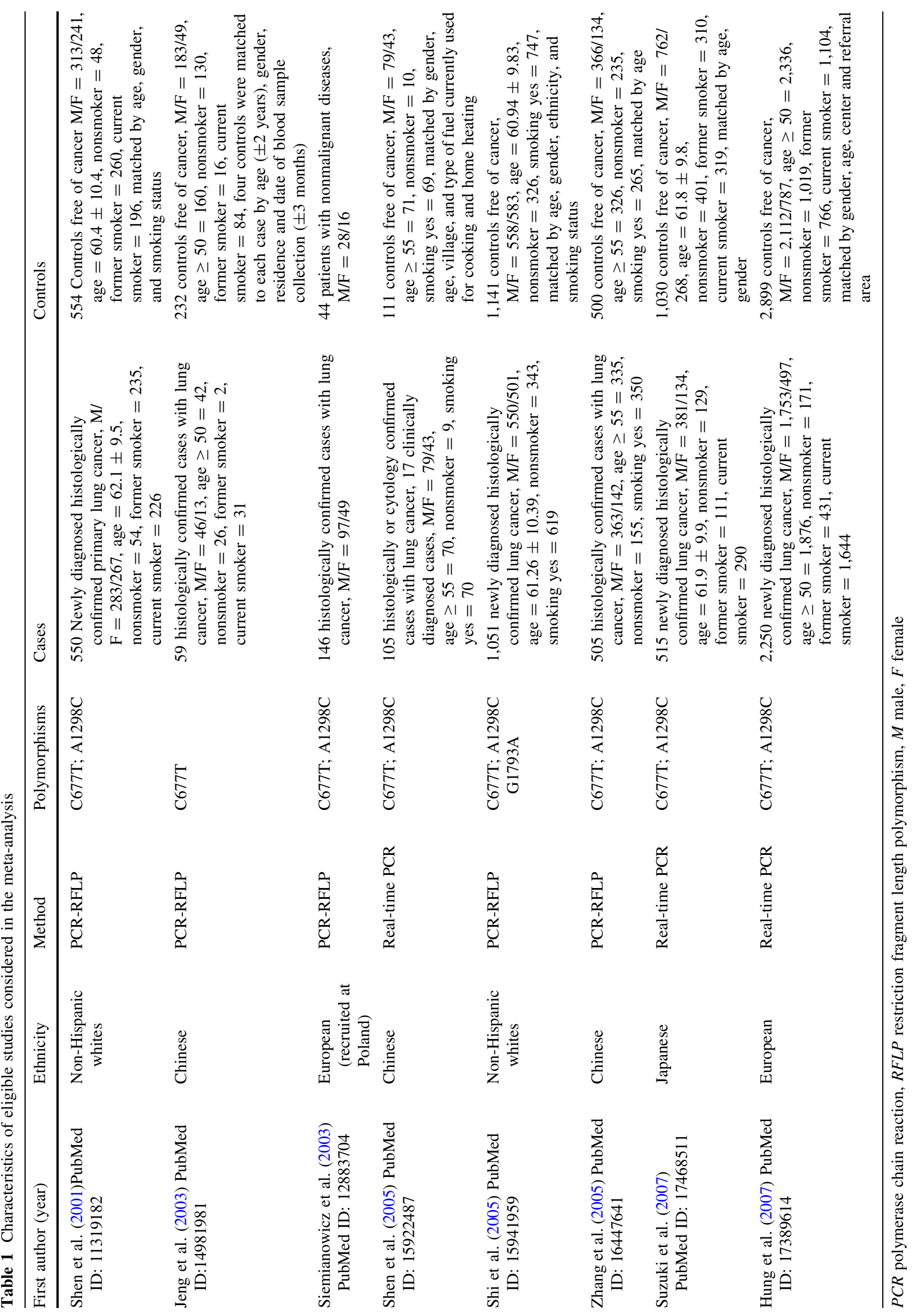




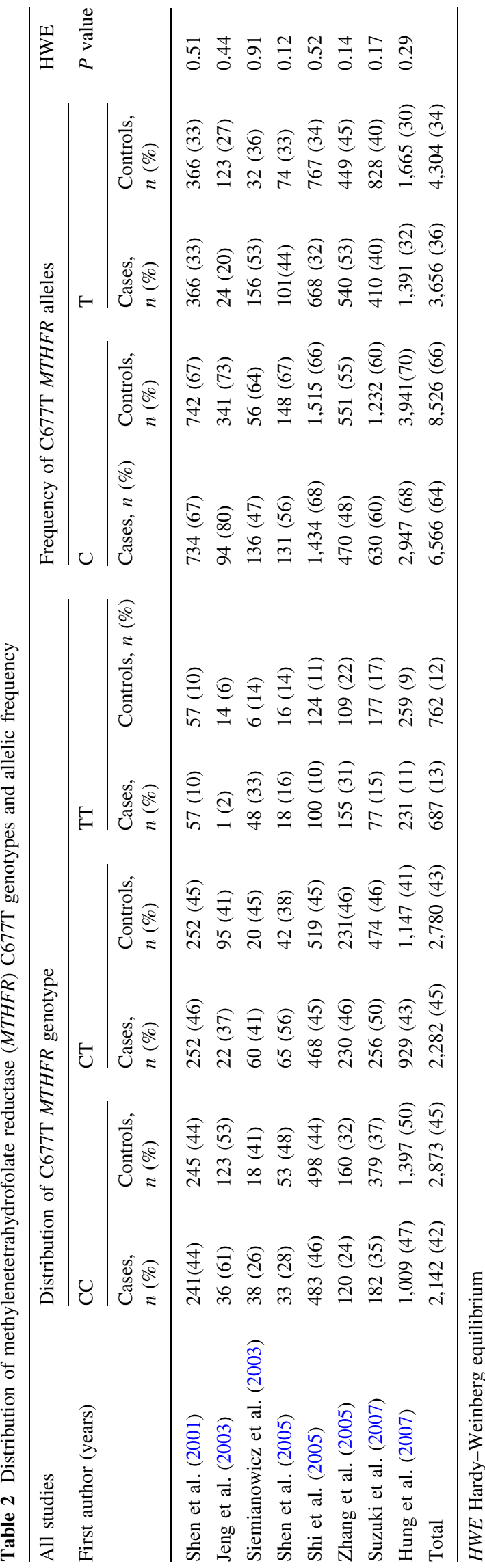

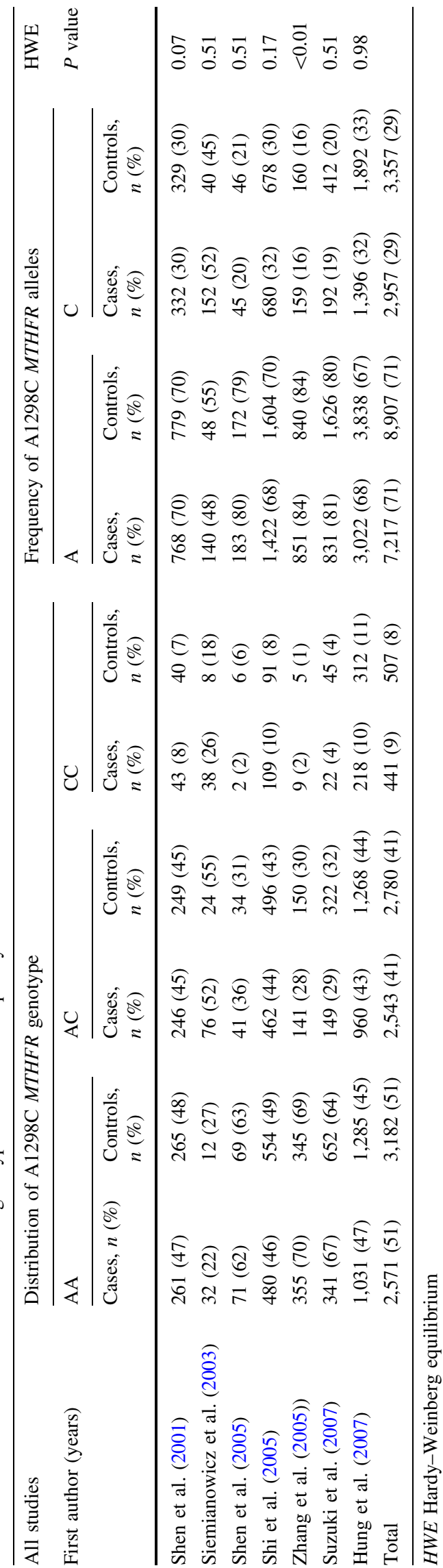


for $\mathrm{C677T}$ of the control group in each study revealed that the studies could not cluster together according to their ethnicity (Fig. 1). The RE model showed that the 677T allele was not associated with the risk of lung cancer compared with the $\mathrm{C}$ allele $[\mathrm{OR}=1.1295 \% \mathrm{CI}$ in parenthesis (0.97-1.28), Fig. 2]. In subgroup analysis, the association was also not significant: OR $=1.14$ (1.0.861.52) for Asians; OR = 1.08 (1.0.91-1.28) for Europeans. The contrast of homozygotes (TT vs. CC), recessive model,

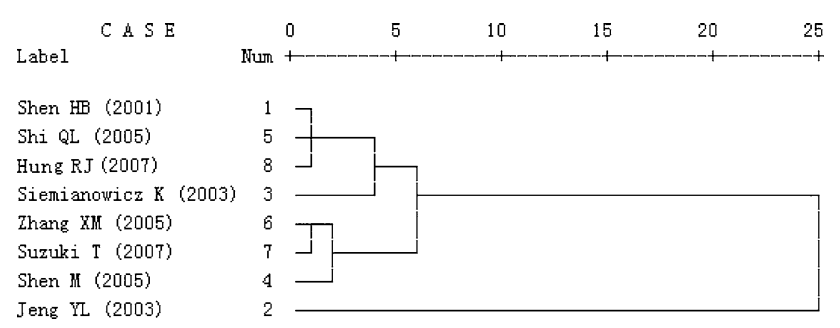

Fig. 1 Hierarchical cluster analysis based on the genotype frequency for C677T and A1298C of the control group in each study dominant model produced the same pattern of results as the allele contrast (Table 4).

In terms of the A1298C polymorphism, there was no heterogeneity among the seven studies comparing the $\mathrm{C}$ vs. the A allele $\left(P=0.24, I^{2}=24.4 \%\right)$, but no significant association was detected by the RE model or the FE model [FE OR $=0.99$ (0.93-1.05); RE OR = 1.00 (0.92-1.08), Fig. 3]. The contrast of homozygotes (TT vs. CC), recessive model, dominant model produced the same pattern of results as the allele contrast (Table 4). The associations of the A1298C polymorphisms with lung cancer did not change during the sensitivity analysis.

Publication bias

Overall, the cumulative and recursive meta-analyses for the allelic contrast (C677T T vs. C) showed that RE pooled OR had a upward trend in the period 2001-2007 (OR $=1.01$ in 2001, first study; OR $=1.18$ in 2003; OR $=1.09$ in 2005; $\mathrm{OR}=1.08$ in 2007). There was no statistical difference between the OR (1.01) of the first study vs. the pooled RE

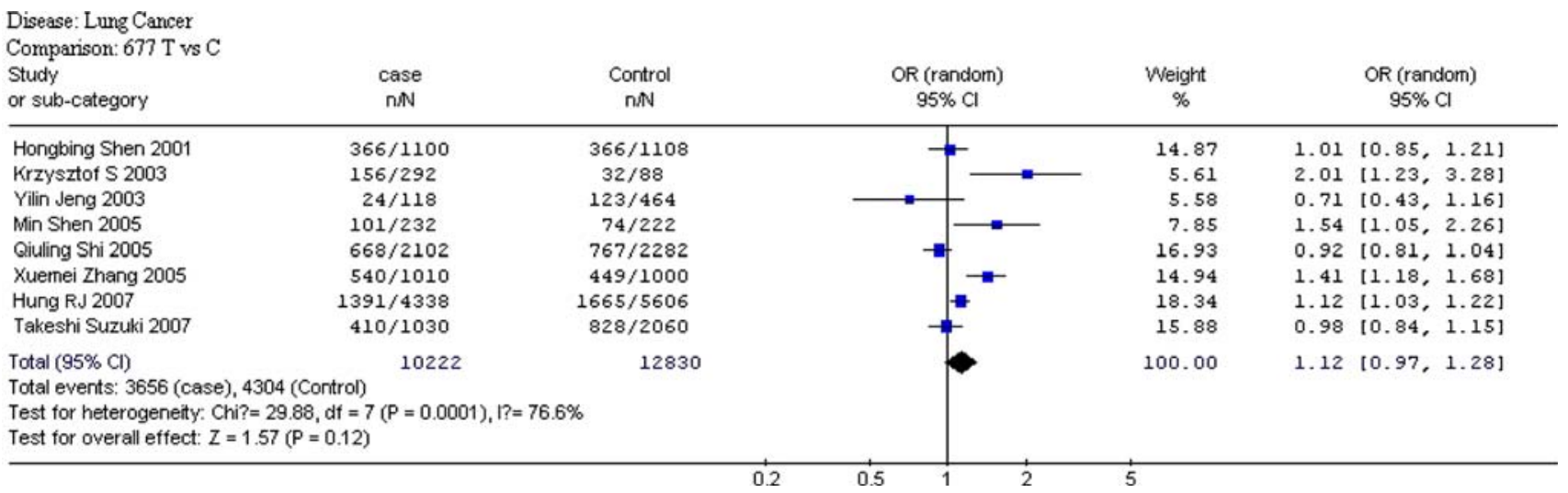

Fig. 2 Overall meta-analysis for C677T polymorphism (T vs. C allele) in lung cancer. Point estimates of the odds ratio (OR) for each study and the accompanying $95 \%$ confidence interval (C)I values obtained with a random effects model are shown

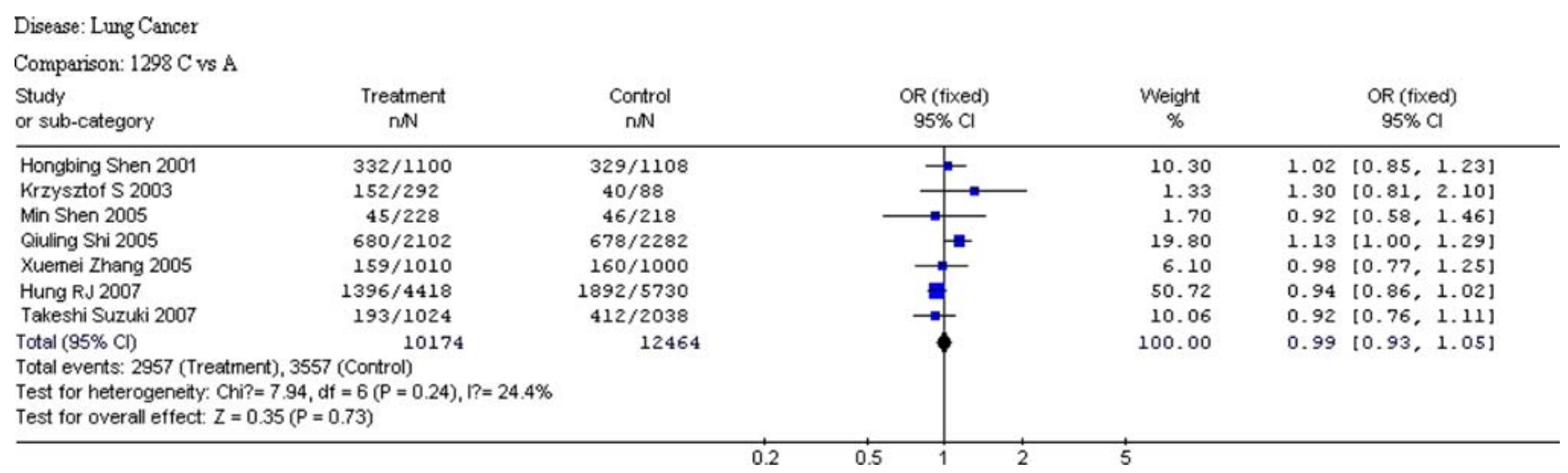

Fig. 3 Overall meta-analysis for A1298C polymorphism (C vs. A allele) in lung cancer. Point estimates of the odds ratio (OR) for each study and the accompanying $95 \%$ confidence interval $(\mathrm{CI})$ values obtained with a fixed effects model are shown 
Table 4 Odds ratios and heterogeneity results for the genetic contrasts of methylenetetrahydrofolate reductase (MTHFR) gene C677T and A1298C polymorphisms for lung cancer patients

\section{$O R$ odds ratio}

a Asian is composed of Chinese and Japanese

${ }^{b}$ European is composed of nonHispanic whites and Europeans

\begin{tabular}{|c|c|c|c|c|c|}
\hline & Population & $\begin{array}{l}\text { Fixed effects OR } \\
(95 \% \text { CI })\end{array}$ & $\begin{array}{l}\text { Random effects OR } \\
(95 \% \mathrm{CI})\end{array}$ & $I^{2}(\%)$ & $\begin{array}{l}P \text { value } \\
Q \text { test }\end{array}$ \\
\hline \multicolumn{6}{|c|}{ Contrast for allele $\mathrm{T}$ in $\mathrm{C} 677 \mathrm{~T}$} \\
\hline \multirow[t]{3}{*}{ Alleles } & All & $1.08(1.02-1.14)$ & $1.12(0.97-1.28)$ & 76.6 & 0.0001 \\
\hline & $\operatorname{Asian}^{\mathrm{a}}$ & $1.15(1.03-1.28)$ & $1.14(0.86-1.52)$ & 80.2 & 0.002 \\
\hline & European $^{\mathrm{b}}$ & $1.06(0.99-1.13)$ & $1.08(0.91-1.28)$ & 77.0 & 0.005 \\
\hline \multirow[t]{3}{*}{ TT to $\mathrm{CC}$} & All & $1.16(1.03-1.31)$ & $1.22(0.92-1.61)$ & 71.6 & 0.0009 \\
\hline & Asian & $1.27(1.02-1.58)$ & $1.26(0.71-2.25)$ & 76.5 & 0.005 \\
\hline & European & $1.12(0.96-1.29)$ & $1.15(0.82-1.62)$ & 71.9 & 0.01 \\
\hline \multirow[t]{3}{*}{ TT to $(\mathrm{CT}+\mathrm{CC})$} & All & $1.11(0.99-1.24)$ & $1.11(0.88-1.40)$ & 64.4 & 0.006 \\
\hline & Asian & $1.14(0.94-1.37)$ & $1.07(0.66-1.72)$ & 73.1 & 0.01 \\
\hline & European & $1.09(0.95-1.26)$ & $1.11(0.83-1.48)$ & 64.0 & 0.04 \\
\hline \multirow[t]{3}{*}{$\mathrm{TT}+\mathrm{CT}$ To CC } & All & $1.10(1.02-1.19)$ & $1.15(0.98-1.36)$ & 68.6 & 0.002 \\
\hline & Asian & $1.24(1.06-1.45)$ & $1.28(0.89-1.84)$ & 74.8 & 0.008 \\
\hline & European & $1.07(0.98-1.16)$ & $1.06(0.90-1.26)$ & 61.8 & 0.05 \\
\hline \multicolumn{6}{|c|}{ Contrast for allele C in A1298C } \\
\hline \multirow[t]{3}{*}{ Alleles } & All & $0.99(0.93-1.05)$ & $1.00(0.92-1.08)$ & 24.4 & 0.24 \\
\hline & Asian & $0.94(0.81-1.08)$ & $0.94(0.81-1.08)$ & 0 & 0.91 \\
\hline & European & $1.00(0.94-1.07)$ & $1.03(0.92-1.17)$ & 57.8 & 0.07 \\
\hline \multirow[t]{3}{*}{$\mathrm{CC}$ to $\mathrm{AA}$} & All & $1.01(0.88-1.16)$ & $1.07(0.84-1.38)$ & 43.5 & 0.10 \\
\hline & Asian & $0.95(0.61-1.48)$ & $0.95(0.49-1.83)$ & 29.7 & 0.24 \\
\hline & European & $1.02(0.87-1.18)$ & $1.12(0.83-1.51)$ & 61.1 & 0.05 \\
\hline \multirow[t]{3}{*}{$\mathrm{CC}$ to $(\mathrm{AC}+\mathrm{AA})$} & All & $1.02(0.89-1.17)$ & $1.08(0.86-1.34)$ & 36.7 & 0.15 \\
\hline & Asian & $0.97(0.62-1.52)$ & $0.96(0.48-1.94)$ & 36.3 & 0.21 \\
\hline & European & $1.03(0.89-1.18)$ & $1.10(0.85-1.43)$ & 52.5 & 0.10 \\
\hline \multirow[t]{3}{*}{$\mathrm{CC}+\mathrm{AC}$ to $\mathrm{AA}$} & All & $0.98(0.91-1.06)$ & $0.98(0.91-1.06)$ & 0 & 0.55 \\
\hline & Asian & $0.95(0.81-1.12)$ & $0.95(0.81-1.12)$ & 0 & 0.69 \\
\hline & European & $0.99(0.91-1.08)$ & $1.01(0.90-1.13)$ & 25.3 & 0.26 \\
\hline
\end{tabular}

OR (1.09) of the subsequent studies. The cumulative and recursive meta-analyses for the allelic contrast (A1298C C vs. A) showed that RE pooled OR remained nonsignificant and fairly constant over the period 2001-2007.

\section{Discussion}

Shen et al. first examined the association between the MTHFR gene polymorphisms and the risk of lung cancer (Shen et al. 2001). After that, the case-control studies provided controversial results. In 2007, two papers also got distinct results (Suzuki et al. 2007; Hung et al. 2007). To clarify this question, we collected all the studies in PubMed and carried out a meta-analysis to examine the association of MTHFR C677T and A1298C polymorphisms with susceptibility to lung cancer. No significant associations were detected for C677T and A1298C. Our current pooled data suggest no evidence for a major role of these two variants in carcinogenesis of lung cancer. Sensitivity analyses indicated that the estimate of the summary effect was robust and did not alter materially when individual studies were excluded. The strength of our analysis is based on the aggregation of published case-control studies; thus, there is more information for investigating the effect of the alleles than in the individual studies (Muncer 2002). The power (1-beta) of this meta-analysis is 93.9 to C677T, which manifests the truth that studied subject size $(5,111 / 6,415$ case and controls) was relatively good.

Heterogeneity for C677T among the eight studies was extreme. Cluster analysis based on genotype frequency for C677T of the control group in each study revealed that the studies could not cluster together according to ethnicity. Although the control group was different from the same ethnical group, its genotype frequency for C677T should assemble in the same ethnical group. Control groups of Asian (Jeng et al. 2003) and European ethnicity (Hung et al. 2007) irrationally cluster together. The following reasons may be the possible source of the heterogeneity: (1) there is a wide variation in the MTHFR $677 \mathrm{~T}$ allele frequency across different populations [18.6-54.5\% in European, from $10.6 \%$ to $44.9 \%$ in American, and from $0.0 \%$ to $9.4 \%$ in African (Spiridonova et al. 2004)], and the frequency has some overlap in different populations; 
(2) Asian and European ethnicity are extremely extensive. Another possible reason is that the large variation in the polymorphic allele frequencies reported among different ethnicities may be actually small, but the real background for that variation may be derived from difficulty for accurate genotyping.

Folate is one of the micronutrients that provide protection against lung carcinogenesis (Bandera et al. 1997; Voorrips et al. 2000; Shen et al. 2003). The MTHFR enzyme plays a pivotal role in folate metabolism, catalyzing the irreversible conversion of 5,10-methylene-THF to 5-methyl-THF. 5,10-methylene-THF involves in purine synthesis and as a substrate by the thymidylate synthetase (TS) enzyme in the methylation of 2'-deoxyuridine 5'monophosphate (dUMP) to deoxythymidine monophosphate (dTMP) (Kim 1999). Reduced availability of dTMP results in misincorporation of uracil into DNA, repair of which may lead to double-strand DNA breaks, which have carcinogenic effects (Blount et al. 1997). 5-methyl-THF is the methyl group donor for the remethylation of homocysteine to methionine, which is subsequently used for DNA methylation, abnormalities of which are also known to play a role in carcinogenesis (Choi et al. 2002). Reduced MTHFR activity would result in increased availability of 5,10-methylene-THF for DNA synthesis and decreased availability of 5-methyl-THF for DNA methylation. Based on this knowledge, MTHFR can act as both the risk and protect factor in carcinogenesis. This point has been supported by the meta-analysis of MTHFR polymorphisms and risks for cancers. MTHFR can reduce the risk of colorectal cancer (Hubner et al. 2007; Huang et al. 2007) and leukemia (Zintzaras et al. 2006a, b) and increase the risk of gastric cancer (Zintzaras 2006). This meta-analysis suggests no association between MTHFR and lung cancer. Taken together, the results imply that different tumors evolve by different pathological pathways and the roles of MTHFR in cancer is determined by its target genes. The latter should be emphasized in future research.

In conclusion, pooled analysis of data from eight (C677T) or seven (A1298C) articles indicates that the MTHFR 677T and 1298C alleles have no association with lung cancer. Although MTHFR polymorphisms associated with the risk of colorectal cancer, leukemia, and gastric cancer were supported by other meta-analysis, our pooled data suggest no evidence for a major role of these two variants in carcinogenesis of lung cancer. The results implied that different tumors evolve by different pathological pathways and the roles of MTHFR in cancer is determined by its target genes.

Acknowledgments This research was supported by the $\mathrm{Ph} . \mathrm{D}$. Program Foundation (No. 20040226001) of MOE.

\section{References}

Amos CI, Xu W, Spitz MR (1999) Is there a genetic basis for lung cancer susceptibility? Recent Results Cancer Res 151:3-12

Bandera EV (1997) Diet and alcohol consumption and lung cancer risk in the New York State Cohort (United States). Cancer Causes Control 8:828-840

Banerjee I, Gupta V, Ganesh S (2007) Association of gene polymorphism with genetic susceptibility to stroke in Asian populations: a meta-analysis. J Hum Genet 52:205-219

Bilello KS, Murin S, Matthay RA (2002) Epidemiology, etiology, and prevention of lung cancer. Clin Chest Med 23:1-25

Blount BC, Mack MM, Wehr CM, MacGregor JT, Hiatt RA, Wang G, Wickramasinghe SN, Everson RB, Ames BN (1997) Folate deficiency causes uracil misincorporation into human DNA and chromosome breakage: implications for cancer and neuronal damage. Proc Natl Acad Sci USA 94:3290-3295

Casas JP, Hingorani AD, Bautista LE, Sharma P (2004) Metaanalysis of genetics studies in ischemic stroke. Thirty-two genes involving approximately 18,000 cases and 58,000 controls. Arch Neurol 61:1652-1662

Choi SW, Mason JB (2002) Folate status: effects on pathways of colorectal carcinogenesis. J Nutr 132:2413-2418

Dichgans M, Markus HS (2005) Genetic association studies in stroke: methodological issues and proposed standard criteria. Stroke 36:2027-2031

Egger M, Davey SG, Schneider M, Minder C (1997) Bias in metaanalysis detected by a simple, graphical test. BMJ 315:629-634

Fontham ET, Correa P, Reynolds P, Wu-Williams A, Buffler PA, Greenberg RS (1994) Environmental tobacco smoke and lung cancer in nonsmoking women. JAMA 271:1752-1759

Hassan A, Markus HS (2000) Genetics and ischemic stroke. Brain 123:1784-1812

Higgins JP, Thompson SG (2002) Quantifying heterogeneity in a meta-analysis. Stat Med 21:1539-1558

Huang Y, Han S, Li Y, Mao Y, Xie Y (2007) Different roles of MTHFR C677T and A1298C polymorphisms in colorectal adenoma and colorectal cancer: a meta-analysis. J Hum Genet 52(1):73-85

Hubner RA, Houlston RS (2007) MTHFR C677T and colorectal cancer risk: a meta-analysis of 25 populations. Int $\mathrm{J}$ Cancer 120(5):1027-1035

Hung RJ, Hashibe M, McKay J, Gaborieau V, Szeszenia-Dabrowska N, Zaridze D, Lissowska J, Rudnai P, Fabianova E, Mates I, Foretova L, Janout V, Bencko V, Chabrier A, Moullan N, Canzian F, Hall J, Boffetta P, Brennan P (2007) Folate-related genes and the risk of tobacco-related cancers in Central Europe. Carcinogenesis 28:1334-1340

Jemal A, Thomas A, Murray T, Thun M (2002) Cancer statistics. CA Cancer J Clin 52:23-47

Jeng YL, Wu MH, Huang HB, Lin WY, You SL, Chu TY, Chen CJ, Sun CA (2003) The methylenetetrahydrofolate reductase 677C $\rightarrow \mathrm{T}$ polymorphism and lung cancer risk in a Chinese population. Anticancer Res 23:5149-5152

Kim YI (1999) Folate and carcinogenesis: evidence, mechanisms, and implications. J Nutr Biochem 10:66-88

Kluijtmans LA, Kastelein JJ, Lindemans J, Boers GH, Heil SG, Bruschke AV, Jukema JW, van den Heuvel LP, Trijbels FJ, Boerma GJ, Verheugt FW, Willems F, Blom HJ (1997) Thermolabile methylenetetrahydrofolate reductase in coronary artery disease. Circulation 96:2573-2577

Lau J, Ioannidis JP, Schmid CH (1997) Quantitative synthesis in systematic reviews. Ann Intern Med 127:820-826

Minna JD, Roth JA, Gazdar AF (2002) Focus on lung cancer. Cancer Cell 1:49-52 
Muncer S (2002) Response to: "power dressing and metaanalysis: incorporating power analysis into meta-analysis. J Adv Nurs 38:274-280

Schwartz AG (2006) Lung cancer: family history matters. Chest 130:936-937

Shen H, Wei Q, Pillow PC, Amos CI, Hong WK, Spitz MR (2003) Dietary folate intake and lung cancer risk in former smokers: a case-control analysis. Cancer Epidemiol Biomarkers Prev 12:980-986

Shen H, Spitz MR, Wang LE, Hong WK, Wei Q (2001) Polymorphisms of methylene-tetrahydrofolate reductase and risk of lung cancer: a case-control study. Cancer Epidemiol Biomarkers Prev 10:397-401

Shen M, Rothman N, Berndt SI, He X, Yeager M, Welch R, Chanock S, Caporaso N, Lan Q (2005) Polymorphisms in folate metabolic genes and lung cancer risk in Xuan Wei, China. Lung Cancer 49:299-309

Shi Q, Zhang Z, Li G, Pillow PC, Hernandez LM, Spitz MR, Wei Q (2005) Sex differences in risk of lung cancer associated with methylene-tetrahydrofolate reductase polymorphisms. Cancer Epidemiol Biomarkers Prev 14:1477-84

Siemianowicz K, Gminski J, Garczorz W, Slabiak N, Goss M, Machalski M, Magiera-Molendowska H (2003) Methylenetetrahydrofolate reductase gene C677T and A1298C polymorphisms in patients with small cell and non-small cell lung cancer. 10:1341-1344

Spiridonova MG, Stepanov WA (2004) Population study of frequency of methylenetetrahydrofolate reductase C677T gene polymorphism in Yakutia. Russ J Genet 40:570-573
Steinmetz KA et al (1991) Vegetables, fruit, and cancer. II. Mechanisms. Cancer Causes Control 2:427-442

Suzuki T, Matsuo K, Hiraki A, Saito T, Sato S, Yatabe Y, Mitsudomi T, Hida T, Ueda R, Tajima K (2007) Impact of one-carbon metabolism-related gene polymorphisms on risk of lung cancer in Japan: a case control study. Carcinogenesis 28:1718-1725

Takezaki T, Hirose K, Inoue M, Hamajima N, Yatabe Y, Mitsudomi T, Sugiura T, Kuroishi T, Tajima K (2001) Dietary factors and lung cancer risk in Japanese: with special reference to fish consumption and adenocarcinomas. Br J Cancer 84:1199-1206

Voorrips LE, et al (2000) A prospective cohort study on antioxidant and folate intake and male lung cancer risk. Cancer Epidemiol Biomarkers Prev 9:357-365

Zhang XM, Miao XP, Tan W, Qu SN, Sun T, Zhou YF, Lin DX (2005) Association between genetic polymorphisms in methylenetetrahydrofolate reductase and risk of lung cancer. Acta Acad Med Sin 27:700-703

Zintzaras E (2006) Association of methylenetetrahydrofolate reductase (MTHFR) polymorphisms with genetic susceptibility to gastric cancer: a meta-analysis. J Hum Genet 51:618-624

Zintzaras E, Ioannidis JP (2005) Heterogeneity testing in metaanalysis of genome searches. Genet Epidemiol 28:123-137

Zintzaras E, Stefanidis I, Santos M, Vidal F (2006a) Do alcoholmetabolizing enzyme gene polymorphisms increase the risk of alcoholism and alcoholic liver disease? Hepatology 43:127-132

Zintzaras E, Koufakis T, Ziakas PD, Rodopoulou P, Giannouli S, Voulgarelis M (2006b) A meta-analysis of genotypes and haplotypes of methylenetetrahydrofolate reductase gene polymorphisms in acute lymphoblastic leukemia. Eur J Epidemiol $21: 885-886$ 